\title{
An efficient SCNT technology for the establishment of personalized and public human pluripotent stem cell banks
}

\author{
Jeoung Eun Lee ${ }^{1}$, Young Gie Chung ${ }^{2}$, Jin Hee Eum ${ }^{1}$, Yumie Lee ${ }^{2}$ E Dong Ryul Lee ${ }^{1,2,3, *}$ \\ ${ }^{1}$ CHA Stem Cell Institute, CHA University, Seongnam 13488, Korea, ${ }^{2}$ Research Institute for Stem Cell Research, CHA Health Systems, Los \\ Angeles, CA 90036, USA, ${ }^{3}$ Department of Biomedical Science, CHA University, Seongnam 13488, Korea
}

\begin{abstract}
Although three different research groups have reported successful derivations of human somatic cell nuclear transfer-derived embryonic stem cell (SCNT-ESC) lines using fetal, neonatal and adult fibroblasts, the extremely poor development of cloned embryos has hindered its potential applications in regenerative medicine. Recently, however, our group discovered that the severe methylation of lysine 9 in Histone $\mathrm{H} 3$ in a human somatic cell genome was a major SCNT reprogramming barrier, and the overexpression of KDM4A, a H3K9me3 demethylase, significantly improved the blastocyst formation of SCNT embryos. In particular, by applying this new approach, we were able to produce multiple SCNT-ES cell lines using oocytes obtained from donors whose eggs previously failed to develop to the blastocyst stage. Moreover, the success rate was closer to $25 \%$, which is comparable to that of IVF embryos, so that our new human SCNT method seems to be a practical approach to establishing a pluripotent stem cell bank for the general public as well as for individual patients. [BMB Reports 2016; 49(4): 197-198]
\end{abstract}

Embryonic stem cells (ESCs) generated using somatic cell nuclear transfer (SCNT) can provide a research model for studying the mechanism of diseases and also provide a patient specific-cell therapy strategy for the treatment of incurable diseases. Recently, several independent research groups have

*Corresponding author. E-mail: drleedr@cha.ac.kr

http://dx.doi.org/10.5483/BMBRep.2016.49.4.055

Received 14 March 2016

Keywords: ESC derivation, HLA-matched, Pluripotent stem cells, Somatic cell nuclear transfer, Stem cell bank, Therapeutic cloning

Abbreviations: H3K9me3, histone H3 lysine 9 trimethylation; HLA, human leukocyte antigen; iPS, induced pluripotent stem cell; SCNT, somatic cell nuclear transfer

Perspective to: Young Gie Chung et al (2015), Histone Demethylase Expression Enhances Human Somatic Cell Nuclear Transfer Efficiency and Promotes Derivation of Pluripotent Stem Cells, Cell Stem Cell, 17(6):758-66. doi: 10.1016/j.stem.2015.10.001 reported the establishment of human SCNT-ESC (Tachibana et al. (2013) Cell 153(6):1228-38. doi: 10.1016/j.cell.2013.05. 006; Chung et al. (2014) Cell Stem Cell 14(6):777-80. doi: 10.1016/j.stem.2014.03.015; Yamada et al. (2014) Nature 510(7506):533-6. doi: 10.1038/nature13287). However, reprogramming human somatic cells through SCNT is painstakingly difficult since only extremely low number of SCNT embryos develop to the blastocyst stage. After a thorough comparative transcriptomic and epigenomic analyses of mouse SCNT embryos and in vitro fertilized (IVF) embryos, Zhang and his colleagues identified one of the intrinsic epigenetic barriers, $\mathrm{H} 3 \mathrm{~K} 9 \mathrm{me} 3$, which prevented the somatic cell nuclear reprogramming and SCNT embryo development (Matoba et al. (2014) Cell 159(4):884-95. doi: 10.1016/j.cell. 2014.09.055). In order to resolve the reprograming barrier, they injected mRNA coding $\mathrm{H} 3 \mathrm{~K} 9 \mathrm{me} 3$ demethylase (mouse $\mathrm{Kdm} 4 \mathrm{~d}$ ) during SCNT procedure, resulting in a significantly increased efficiency of cloned embryonic development. Subsequently, Zhang's group and our group have performed comparative transcriptomic and epigenomic analyses of human IVF and SCNT embryos, and found that H3K9me3 was also a major barrier for human SCNT reprogramming.

After we applied a similar strategy for human SCNT, an injection of human KDM4A mRNA into reconstituted human eggs, the treated group showed very similar transcriptional pattern to their 8-cell stage IVF counterparts during the embryonic genome activation (EGA), and a significantly higher developmental rate of blastocysts than untreated control group. Using this improved SCNT method, we successfully established multiple functionally normal SCNT-ESC lines, whilst none of the control (mRNA-non-injected) embryos were able to establish an ESC line. More importantly, establishment of SCNT-ESC in the study was not limited to a particular oocyte donor. In fact, it is well understood that only oocytes from certain specific females can support the development of SCNT embryos to the blastocyst stage (Tachibana et al. (2013) Cell 153(6):1228-38. doi: 10.1016/j.cell.2013.05.006; Chung et al. (2014) Cell Stem Cell 14(6):777-80. doi: 10.1016/j.stem.2014. 03.015). To prove that our new procedure could overcome the egg donor variation, we intentionally used oocytes from donors whose eggs previously failed to develop to blastocysts. Therefore, the introduction of KDM4A into cloned eggs 
eliminates the reliance on finding specific donors, resulting in a bigger egg donor pool.

The crux of regenerative medicine rests on transplanted cells being accepted (i.e., not rejected) by the patient's immune system. To facilitate this, the use of autologous pluripotent stem cell (PSC) obtained from SCNT or induced pluripotent stem cell (iPSC) technology is theoretically the ideal candidate to avoid or minimize host immune responses. However, establishing autologous PSC for each and every individual would be an impractical, time consuming and technically difficult task. Therefore, focusing on establishing a cell bank with homozygous HLA genotyped PSCs would be the best feasible and practical alternative. This type of cells could be transplanted to patients whose only one half of HLA (haplotype) is matched resulting in an exponential number of people benefitting. Already research teams from the UK and Japan have reported that 150 and 140 HLA-homozygous-iPSCs could match $93 \%$ of the UK and $90 \%$ of the Japanese population.

Unfortunately, the use of iPSCs for any therapeutic application is still in doubt due to the intrinsic defect in nuclear reprogramming as reflected by variability of cell lines. Thus, it is the general consensus that SCNT derived stem cells more resemble their IVF embryo derived ES cells than iPS cells, as revealed by global gene expression profiling and functional studies. However, SCNT ES cells have not been used widely until recently because of the extremely low success rate. By virtue of recent advent of RNA sequencing technology and gene expression profiling technology, we and other research groups were not only able to produce human SCNT ES cells but also significantly improved ES cell derivation rate comparable to that of regular IVF embryos. This leads us to suggest that this technology would be instrumental to establishing PSC banks with homozygous HLA genotypes. Moreover, the SCNT ES cells house the fresh young mitochondria derived from the oocytes, thus it can be used to treat certain diseases related to defective mitochondria.

In our recent study, we developed a very efficient human SCNT ES cell derivation method as evidenced by producing multiple SCNT-ES cell lines from healthy subjects of varying ages, and from patients with several types of diseases. Therefore, we believe our methodology can be used to successfully establish a PSC cell banks with homozygous HLA genotype which is the most hopeful step towards unparalleled therapeutic potentials for the general public.

\section{ACKNOWLEDGEMENTS}

This work was supported by grants from the Bio \& Medical Technology Development Program (2012M3A9C6049723 and 2015M3A9C6028961) of NRF and MSIP of Republic of Korea. 